\title{
CONCERNING THE BOUNDARIES OF DOMAINS OF A CONTINUOUS CURVE*
}

\author{
BY W. L. AYRES
}

We shall consider a space $M$ consisting of all the points of a plane continuous curve $M \dagger$, and all point sets mentioned are assumed to be subsets of $M$. A connected set of points $D$ of $M$ is said to be an $M$-domain if $M-D$ is closed. The set of all limit points of $D$ which do not belong to $D$ is called the $M$-boundary of $D$. If $D$ is an $M$-domain, $D^{\prime}$ denotes the $M$-boundary of $D$, and $\bar{D}$ denotes the set $D+D^{\prime}$. The $M$-boundary of an $M$-domain $D$ is closed but not necessarily connected, even if $D$ is simply connected, as we may easily show by examples. If $N$ is a continuum, a maximal connected subset of $M-N$ is called a complementary $M$-domain of $N$.

THEOREM I. $\ddagger$ Every closed and connected subset of the $M$-boundary of a complementary $M$-domain of a continuous curve $N$ is a continuous curve.

Proof. Let $K$ denote a closed and connected subset of the $M$-boundary of a complementary $M$-domain $D$ of $N$. Suppose $K$ is not connected im kleinen. Then there exist $\$$ two concentric circles $C_{1}$ and $C_{2}$ (let $r_{i}$ denote the radius of $C_{i}$ and let $\left.r=r_{1}-r_{2}>0\right)$ and an infinite sequence of subcon-

* Presented to the Society, February 26, 1927.

$\dagger$ A point set which is closed, connected and connected im kleinen is called a continuous curve. In general it may be either bounded or unbounded.

$\ddagger$ For the case where $M$ is the entire plane and $N$ is bounded, see R. L. Wilder, Concerning continuous curves, Fundamenta Mathematicae, vol. 7 (1925), p. 361.

$\S$ See R. L. Moore, Report on continuous curves from the viewpoint of analysis situs, this Bulletin, vol. 29 (1923), p. 296. This theorem is stated by Moore for a bounded continuum, but the theorem remains true without the condition of boundedness. 
tinua of $K, K_{\infty}, K_{1}, K_{2}, K_{3}, \cdots$, such that (1) each of these continua $K_{\alpha}(\alpha=\infty, 1,2, \cdots)$ contains a point $a_{\alpha}$ on $C_{1}$ and a point $b_{\alpha}$ on $C_{2}$, but no point exterior to $C_{1}$ or interior to $C_{2},(2)$ no two of these continua have a point in common and no one of them, except possibly $K_{\infty}$, is a proper subset of any connected subset of $K$ which contains no point without $C_{1}$ or within $C_{2}$, (3) the set $K_{\infty}$ is the sequential limiting set of the sequence of continua $K_{1}, K_{2}, K_{3}, \ldots$. For any $i$, let $K_{i}{ }^{*}=K_{\infty}+K_{i+1}+K_{i+2}+\cdots$. Let $d_{1}$ be the smaller of the numbers $\frac{1}{4} r$ and $\frac{1}{2} d\left(K_{1}, K_{1}^{*}\right) \dagger$.

Let $R_{1}$ denote the set of all points $[P]$ of $N$ such that the distance from $P$ to some point of $K_{1}$ is less than $d_{1}$. The set $R_{1}$ is an open subset of $N$ and hence $R_{1}$ contains an arc from $a_{1}$ to $b_{1} . \neq$ This arc contains a subarc $x_{1} y_{1}$ such that $x_{1}$ is on $C_{2}$ and $y_{1}$ is on $C_{1}$ and every other point of $x_{1} y_{1}$ is between $C_{1}$ and $C_{2}$.

For each $n>1$, let $d_{n}$ be the smallest of the numbers $d_{n-1}, r 2^{-n-1}$, and $\frac{1}{2} d\left(K_{n}, K_{n}{ }^{*}+x_{1} y_{1}+x_{2} y_{2}+\cdots+x_{n-1} y_{n-1}\right)$. Let $R_{n}$ denote the set of all points $[P]$ of $N$ such that there is some point of $K_{n}$ whose distance from $P$ is less than $d_{n}$. The set $R_{n}$ contains an arc from $a_{n}$ to $b_{n}$ and this arc contains a subarc $x_{n} y_{n}$ such that $x_{n}$ is on $C_{2}, y_{n}$ is on $C_{1}$, and every other point of $x_{n} y_{n}$ is between $C_{1}$ and $C_{2}$.

There exists an increasing sequence of positive integers $n_{1}, n_{2}, n_{3}, \cdots$, such that (1) $C_{2}$ contains three points $X_{1}$, $X_{2}, X_{3}$ such that every point $x_{n_{i}}$ lies on the $\operatorname{arc} X_{1} X_{2} X_{3}$ of $C_{2}$ and in the order $X_{1} X_{2} x_{n_{1}} x_{n_{2}} \cdots X_{3}$, (2) $C_{1}$ contains three points $Y_{1}, Y_{2}, Y_{3}$ such that every point $y_{n_{i}}$ lies on the arc $Y_{1} Y_{2} Y_{3}$ of $C_{1}$ and in the order $Y_{1} Y_{2} y_{n_{1}} y_{n_{2}} \cdots Y_{3}$, (3) $X_{3}$ is the sequential limit point of $\left[x_{n_{i}}\right]$ and $Y_{3}$ is the sequential limit point of $\left[y_{n_{i}}\right]$. Clearly $K_{\infty}$ contains $X_{3}$ and $Y_{3}$.

$\dagger$ If $A$ and $B$ are sets of points, the symbol $d(A, B)$ denotes the lower bound of all numbers $d(x, y)$, where $x$ is a point of $A, y$ is a point of $B$, and $d(x, y)$ is the distance from $x$ to $y$.

$\ddagger$ R. L. Moore, Concerning continuous curves in the plane, Mathematische Zeitschrift, vol. 15 (1922), p. 255. 
The set $K_{\infty}$ contains points $X$ and $Y$ on the circles which are concentric with $C_{1}$ and with radii $r_{2}+r / 10$ and $r_{1}-r / 10$ respectively. Let $\eta$ be the smaller of the two numbers $r / 10$ and $r_{2}$. Since $N$ is connected im kleinen, there exists a positive number $\delta_{\eta}$ such that any point of $N$ within a distance $\delta_{\eta}$ of $X$ or $Y$ may be joined to $X$ or $Y$, as the case may be, by an arc of $N$ every point of which is within a distance $\eta$ of $X$ or $Y$. Let $n_{s}$ be the smallest integer such that $x_{n_{s}} y_{n_{s}}$ contains two points $P$ and $Q$ such that $d(P, X)<\delta_{\eta}$ and $d(Q, Y)<\delta_{\eta}$. Then $N$ contains arcs $P X$ and $Q Y$, every point of which is within a distance $\eta$ of $X$ and $Y$ respectively. Let order be defined on these arcs as from $P$ to $X$ and from $Q$ to $Y$. The arcs $P X$ and $Q Y$ have points in common with every arc $x_{n_{i}} y_{n_{i}}$ for $i \geqq s$.

Let $V_{10}$ and $V_{20}$ be the last points the arcs $P X$ and $Q Y$ have in common with $x_{n_{s}} y_{n_{s}}$. Let $U_{11}$ and $U_{21}$ be the first points and $V_{11}$ and $V_{21}$ be the last points the subarcs $V_{10} X$ and $V_{20} Y$ have in common with $x_{n_{s+1}} y_{n_{s+1}}$. Let $U_{12}$ and $U_{22}$ be the first points the subarcs $V_{11} X$ and $V_{21} Y$ have in common with $x_{n_{s+2}} y_{n_{s+2}}$. The set $J$, composed of the arcs $V_{10} V_{20}$ of $x_{n_{s}} y_{n_{s}}, U_{11} V_{11}$ and $U_{21} V_{21}$ of $x_{n_{s+1}} y_{n_{s+1}}, U_{12} U_{22}$ of $x_{n_{s+2}} y_{n_{s+2}}$, $V_{10} U_{11}$ and $V_{11} U_{12}$ of $P X, V_{20} U_{21}$ and $V_{21} U_{22}$ of $Q Y$, is a simple closed curve. The subarc of $x_{n_{s+1}} y_{n_{s+1}}$ lying within $J$ and the arc $x_{n_{s+3}} y_{n_{s+3}}$ have points $p_{1}$ and $p_{3}$, respectively, in common with the circle concentric with $C_{1}$ and with radius $r_{2}+\frac{1}{2} r$. The point $p_{1}$ is interior to $J$ and within a distance $d_{n_{s+1}}$ of some point of $K$ and this point is a limit point of $D$. Thus $D$ contains a point in the interior of $J$. Similarly $D$ contains a point within a distance $d_{n_{s+3}}$ of $p_{3}$ and thus in the exterior of $J$. Since $D$ is connected $D$ must contain a point of $J$. But as $J$ belongs to $N$ and $D$ to $M-N, D$ cannot contain a point of $J$. Thus the assumption that $K$ is not connected im kleinen has led to a contradiction.

THEOREM II. If a maximal connected subset $K$ of the boundary of an $M$-domain is a continuous curve, every closed and connected subset of $K$ is a continuous curve. 
Definition. If $D$ is an $M$-domain and $P$ is a point of $M-\bar{D}$, the $M$-boundary of the maximal connected subset of $M-\bar{D}$ containing $P$ will be called the $M$-boundary of $D$ with respect to $P$. This is a generalization of the notion of outer boundary as defined by R. L. Moore.* If $M$ is the entire plane, $D$ is bounded, and $P$ is a point of the maximal connected subset of $M-\bar{D}$ which is untounded, then the $M$-boundary of $D$ with respect to $P$ is exactly the outer boundary of $D$ as defined by Moore.*

Theorem III. $\dagger$ If $D$ is an $M$-domain, $P$ is a point of $M-\bar{D}$, and $B$ is the $M$-boundary of $D$ with resłect to $P$, then $B$ is the entire $M$-boundary of some $M$-domain which contains $D$.

Proof. The entire set $D$ lies in the same maximal connected subset of $M-B$ and let $R$ denote this maximal connected subset. Evidently $R$ is an $M$-domain containing $D$. Suppose $Q$ is a point of the $M$-boundary of $R$. If $Q$ does not belong to $B, Q$ belongs to a maximal connected subset of $M-B$ which is different from $R$. Then $Q$ is not a limit point of $R$. $\ddagger$ Therefore every point of the $M$-boundary of $R$ is a point of $B$. Conversely every point of $B$ is an $M$-boundary point of $D$ and thus of $R$. Hence $B$ is identical with the $M$-boundary of $R$, an $M$-domain containing $D$.

Theorem IV. If (1) $D$ is an $M$-domain and $P$ is a point of $M-\bar{D}$, (2) every maximal connected subset of $D^{\prime}$ is a continuous curve, (3) the $M$-boundary of $D$ with respect to $P$, which we denote by $B$, is bounded, then every maximal connected subset of $B$ is either a point, a simple continuous arc or a simple closed curve.

Proof. Let $R$ be the maximal connected subset of $M-\bar{D}$ containing $P$, and let $B_{1}$ be a maximal connected subset of $B$.

* Concerning continuous curves in the plane, loc. cit., p. 256.

$\dagger$ Compare R. L. Moore, Concerning continuous curves in the plane, loc. cit., Theorem 3, p. 258 .

$\ddagger$ See R. L. Moore, A characterization of a continuous curve, Fundamenta Mathematicae, vol. 7 (1925), Lemma 1, p. 302. 
By Theorem II, $B_{1}$ is a continuous curve and $B_{1}$ is bounded by hypothesis. If $B_{1}$ consists of a single point, our theorem is proved. If $B_{1}$ consists of more than a single point then by a theorem due to Mazurkiewicz, ${ }^{*} B_{1}$ contains two points $x$ and $y$ which do not cut $B_{1}$. The continuous curve $B_{1}$ contains an arc $x z y$ from $x$ to $y$. If $B_{1} \equiv x z y$, our theorem is proved. If not, let $p$ be a point of $B_{1}$ which does not lie on $x z y$. By Theorem III, $B$ is the entire $M$-boundary of some $M$-domain $H$ which contains $D$. Clearly $R$ and $H$ are mutually exclusive and $B$ is the entire $M$-boundary of each. By a theorem due to Wilder, $\dagger$ if $p_{1}$ and $p_{2}$ are points of $R$ and $H$ respectively there exist arcs $p_{1} x$ and $p_{1} y$ which lie except for $x$ and $y$ wholly in $R$ and $\operatorname{arcs} p_{2} x$ and $p_{2} y$ which lie except for $x$ and $y$ wholly in $H$. The sets $p_{1} x+p_{1} y$ and $p_{2} x+p_{2} y$ contain arcs $x u y$ and $x v y$ which lie wholly in $R$ and $H$ respectively except for the points $x$ and $y$.

Let $J_{1}, J_{2}, J_{3}$ be the simple closed curves formed of $x u y+x z y, x v y+x z y, x u y+x v y$ respectively and let $I_{i}$ and $E_{i}$ denote the interior and exterior of $J_{i}(i=1,2,3)$. We have three cases to consider:

Case (1). Suppose $I_{3}=I_{1}+I_{2}+\langle x z y\rangle . \ddagger$ Any point $q$ of $B-x z y$ lies either in $I_{1}, I_{2}$ or $E_{3}$. If $q$ lies in $I_{1}, I_{1}$ contains a point of $H$ since $q$ is a limit point of $H$. The exterior $E_{1}$ contains $\langle x v y\rangle$ of $H$. But $H$ is connected and contains no point of $J_{1}$. Hence $I_{1}$ contains no point of $B-x z y$. Similarly $I_{2}$ contains no point of $B-x z y$. Then every point of $B-x z y$ lies in $E_{3}$. Since $x$ and $y$ are not cut-points of $B_{1}$, the continuous curve $B_{1}$ contains an arc $p x$ which does not contain $y$ and an arc $p y$ which does not contain $x$. The set $p x+p y$ contains an arc $x w y$ from $x$ to $y$. Since $p$ is in $E_{3}$ and no point of $J_{3}$ except $x$ and $y$ is a point of $B_{1}$, the set $\langle x w y>$ lies

* Un théorème sur les lignes de Jordan, Fundamenta Mathematicae vol. 2 (1921), pp. 119-130.

$\dagger$ Loc. cit., Theorem 1, p. 342.

$\ddagger$ If $x z y$ denotes a simple continuous arc with end-points $x$ and $y$ $<x z y>$ denotes $x z y-(x+y)$. 
entirely in $E_{3}$, and thus the arcs $x w y$ and $x z y$ have only $x$ and $y$ in common. Let $J_{4}$ be the simple closed curve $x z y+x w y$. We will show that $B-J_{4}$ is vacuous and thus prove $B \equiv B_{1} \equiv J_{4}$. Suppose $B-J_{4}$ contains a point $q_{1}$. If $q_{1}$ lies in the interior of $J_{4}$ both $R$ and $H$ have points in the interior of $J_{4}$ since $q_{1}$ is a limit point of both domains. One of the two sets $\langle x u y\rangle$ or $\langle x v y\rangle$, say $\langle x u y\rangle$, lies entirely in the exterior of $J_{4}$. Then $R$ contains points interior and exterior to $J_{4}$ but contains no point of $J_{4}$, which is impossible. If $q_{1}$ lies in the exterior of $J_{4}$, both $R$ and $H$ contain points in the exterior of $J_{4}$, and one of them contains points in the interior, which is impossible. Therefore $B-J_{4}$ is vacuous, which proves the theorem for this case.

Case (2). Suppose $I_{2}=I_{1}+I_{3}+\langle x u y\rangle$.

Case (3). Suppose $\left.I_{1}=I_{2}+I_{3}+<x v y\right\rangle$.

In Cases (2) and (3), it may be proved by methods similar to those of Case (1) that $B \equiv B_{1}$ and $B_{1}$ is a simple closed curve. Therefore $B_{1}$ is either a point, a simple continuous arc, or a simple closed curve.

In proving Theorem IV we have obtained this result:

THEOREM V. Under the hypothesis of Theorem IV, if any maximal connected subset $J$ of the $M$-boundary of $D$ with respect to $P$ is a simple closed curve, then $J$ is the entire $M$-boundary' of $D$ with respect to $P$.

Theorem VI. If $D$ is an $M$-domain, $P$ is a point of $M-\bar{D}$, $R$ is the maximal connected subset of $M-\bar{D}$ containing $P$, and $Q$ is a point of the maximal connected subset of $M-\bar{R}$ which contains $D$, then $R^{\prime}$ is the $M$-boundary of $R$ with respect to $Q$.

Proof. Let $H$ denote the maximal connected subset of $M-\bar{R}$ which contans $D$. By definition $H^{\prime}$ is the $M$-boundary of $R$ with respect to $Q$. The set $H^{\prime}$ is a subset of $R^{\prime}$ since the $M$-boundary of a domain with respect to a point is always a subset of the $M$-boundary of the domain. By definition, $R^{\prime}$ is the $M$-boundary of $D$ with respect to $P$. Then every point of $R^{\prime}$ is a limit point of $D$ and thus of $H$. As $H$ is a subset of 
$M-\bar{R}$, no point of $R^{\prime}$ is a point of $H$. Therefore every point of $R^{\prime}$ is an $M$-boundary point of $H$, that is, $R^{\prime}$ is a subset of $H^{\prime}$. Hence $R^{\prime} \equiv H^{\prime}$.

The University of Pennsylvania

\section{A THEOREM ON CONNECTED POINT SETS}

BY C. KURATOWSKI AND C. ZARANKIEWICZ

1. Introduction. The purpose of this paper is to prove the following theorem.

Theorem. If $S$ is a connected point set and $Z$ is the set of all points such that $S-p$ is neither connected nor the sum of two connected sets, then $Z$ is finite or countable.

2. Lemma. If $S, P$, and $Q$ are three non-vacuous connected sets (or points), and if

$$
\begin{aligned}
& P+Q \subset S, \\
& A \subset S-P, \\
& A \cdot Q=0,
\end{aligned}
$$

$$
\begin{aligned}
& P \cdot Q=0, \\
& B \subset S-Q, \\
& B \cdot P=0,
\end{aligned}
$$

(7) $A$ and $S-P-A$ are mutually separated,

(8) $B$ and $S-Q-B$ are mutually separated, then $A \cdot B=0$.

Proof. By (1) and (3), $A+P \subset S$. Hence, by (2) and (5), $A+P=(A+P) \cdot(S-Q)$. By (4), $S-Q=B+(S-Q-B)$. Therefore

$$
A+P=(A+P) \cdot B+(A+P) \cdot(S-Q-B) .
$$

It follows from (2) and (6) that $P=P-Q-B \subset(A+P)$ $(S-Q-B)$. Since $P \neq 0$, we have

$$
(A+P) \cdot(S-Q-B) \neq 0 .
$$

Now, by (8), the sets $(A+P) \cdot B$ and $(A+P) \cdot(S-Q-B)$ are mutually separated. On the other hand, by virtue of a 